\title{
Some Estimation Approaches of Intensities for a Two Stage Open Queueing Network
}

\author{
V. K. Gedam ${ }^{1}$ and S. B. Pathare ${ }^{2, *}$ \\ ${ }^{1}$ Department of Statistics, University of Pune, India \\ ${ }^{2}$ Indira College of Commerce and Science, India
}

Received 25 June 2013; Accepted 13 January 2014

Editor: Paulo Canas Rodrigues

\begin{abstract}
In this paper we propose a consistent and asymptotically normal estimator (CAN) for intensity parameters $\rho_{1} \& \rho_{2}$ for a queueing network with distribution-free inter-arrival and service times. Using this estimator and its estimated variance, a 100(1 $\alpha) \%$ asymptotic confidence interval for intensities is constructed. Variance-stabilized bootstrap-t, Bayesian bootstrap, Percentile bootstrap are also applied to develop the confidence intervals for intensities. A comparative analysis is conducted to demonstrate performances of the confidence intervals of intensities for a queueing network with short run.
\end{abstract}

Keywords Coverage percentage; Relative coverage; Variance-stabilized bootstrap-t;

Bayesian bootstrap; Percentile bootstrap; Slutsky's theorem

DOI: $10.19139 /$ soic.v2i1.19

\section{Introduction}

Queueing network is characterized by one or more sources of job arrivals and corresponding one or more sinks that absorb jobs departing from the network. Consider the two-stage open queueing network shown in Figure-1.

The system consists of two nodes with respective service rates $\mu_{1}$ and $\mu_{2}$. The external arrival rate is $\lambda$. Burke (1956) has shown that the output of an M/M/1

\footnotetext{
${ }^{*}$ Correspondence to: Indira College of Commerce and Science,PUNE-411033, Maharashtra, India. E-mail: sureshpathare23@gmail.com
}

ISSN 2310-5070 (online) ISSN 2311-004X (print)

Copyright (c) 2014 International Academic Press 
queue is also Poisson with rate $\lambda$. Traffic intensities are defined as the ratios

$$
\rho_{1}=\frac{\lambda}{\mu_{1}}, \quad \rho_{2}=\frac{\lambda}{\mu_{2}}
$$

where $1 / \lambda$ represent mean inter-arrival time and $1 / \mu_{1}, 1 / \mu_{2}$ denotes mean service times at node- 1 and node- 2 respectively. Intensity parameters $\rho_{1}$ and $\rho_{2}$ can be interpreted as expected number of arrivals per mean service time in the limit, an important parameters called utilization factors that measures the average use of the service facility (Gross and Harris (1998)). The condition for stability of the system is that $\rho_{1}$ and $\rho_{2}$ must be less than unity.

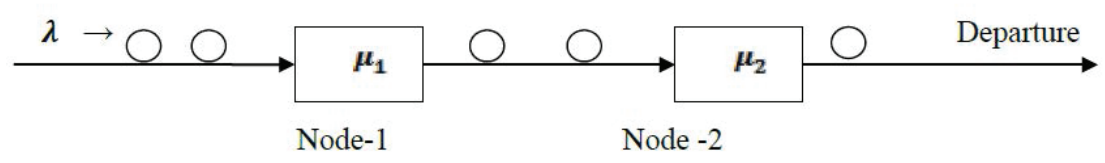

Figure-1: Two-stage open queueing network.

Jackson (1957) showed that the product form solution also applies to open network of Markovian queues with feedback, also Jacksons theorem states that each node behaves like an independent queue. Disney (1975) introduces basic properties of queueing networks. Thiruvaiyaru, Basawa and Bhat (1991) established maximum likelihood estimators of the parameters of an open Jackson network, and their joint asymptotic normality. Thiruvaiyaru and Basawa (1996) considered the problem of estimation for the parameters in a Jacksons type queueing network with the arrival at each node following renewal process and service time distribution being arbitrary. Open queueing networks are useful in studying the behavior of computer communication networks (Kleinrock, 1976).

Efron (1979, 1982, and 1987) originally developed and proposed the bootstrap to estimate the sampling distribution of any statistic. Today the bootstrap becomes the most powerful nonparametric estimation procedure. Based upon the bootstrap resampling technique, most statisticians utilize the standard bootstrap (SB), percentile bootstrap ( $\mathrm{PB})$, and bias-corrected and accelerated bootstrap (BCaB) approaches to produce confidence intervals for practical problems. Rubin (1981) presented the Bayesian bootstrap (BB) technique of resampling. Ke and $\mathrm{Chu}$ (2006) proposed a consistent and asymptotically normal estimator of intensity for a queueing system with distribution-free inter-arrival and service times. Ke and Chu (2009) constructed confidence intervals of intensity for a queueing system, which are based on different bootstrap methods. 
In this paper we propose different types of interval estimations for intensity parameters $\rho_{1}, \rho_{2}$ for a two- stage open queueing network with distributionfree interval and service times. Also, numerical simulation study is conducted to demonstrate performances of the interval estimation approaches for a twostage open queueing network with short run. All simulation results are shown by appropriate tables for illustrating performances of all estimation approaches. Finally some conclusions are made.

\section{Nonparametric Statistical Inference for Estimating Intensity Parameters}

Let $\left(X_{i}, Y_{i}, i=1,2\right)$ be nonnegative random variables representing respectively inter-arrival times and service times at node- 1 and node- 2 of a queueing network.The random variables $\left(X_{i}, Y_{i}, i=1,2\right)$ of node- 1 and node-2 are independent. Consider $\left(X_{i j}, Y_{i j}, i=1,2, j=1,2, \cdots, n\right)$ is a random sample drawn from $\left(X_{i}, Y_{i}, i=1,2\right)$ for $j^{t h}$ customer at $i^{\text {th }}$ node. The intensities are defined as follows:

$$
\rho_{1}=\frac{\mu_{Y_{1}}}{\mu_{X_{1}}} \text { and } \rho_{2}=\frac{\mu_{Y_{2}}}{\mu_{X_{2}}},
$$

where $\mu_{X_{1}}, \mu_{X_{2}}$ denote the mean inter-arrival times at node-1 and node-2 respectively. Also $\mu_{Y_{1}}, \mu_{Y_{2}}$ denote the mean service times at node-1 and node-2 respectively. Let $\left(\bar{X}_{i}, \bar{Y}_{i}, i=1,2\right)$ be the sample means of $\left(X_{i j}, Y_{i j}, i=1,2, j=\right.$ $1,2, \cdots, n)$ respectively. According to the Strong Law of Large Numbers (see Rousses, 1997, p.196), we know that $\left(\bar{X}_{i}, \bar{Y}_{i}, i=1,2\right)$ are strongly consistent estimators of $\left(\mu_{X_{i}}, \mu_{Y_{i}}, i=1,2\right)$ respectively. Thus strongly consistent estimators of intensities are given by

$$
\hat{\rho}_{i}=\frac{\bar{Y}_{i}}{\bar{X}_{i}}, \quad i=1,2 .
$$

The true distributions of $\left(X_{i}, Y_{i}, i=1,2\right)$ are not often known in practice so the exact distributions of $\hat{\rho}_{i}, i=1,2$ cannot be derived. But under the assumption that $X_{i}$ and $Y_{i}$ being independent, the asymptotic distributions of $\hat{\rho}_{i}, i=1,2$ can be developed by the following procedure. By Slutsky's theorem (Hogg \& Craig, 1995), we have

$$
\sqrt{n}\left(\begin{array}{c}
\hat{\rho}_{1}-\rho_{1} \\
\hat{\rho}_{2}-\rho_{2}
\end{array}\right) \stackrel{D}{\rightarrow} N_{2}\left(\left(\begin{array}{l}
0 \\
0
\end{array}\right),\left(\begin{array}{cc}
\sigma_{1}^{2} & 0 \\
0 & \sigma_{2}^{2}
\end{array}\right)\right)
$$

where $\sigma_{i}^{2}=\left(\mu_{X_{i}}^{2} \sigma_{Y_{i}}^{2}+\mu_{Y_{i}}^{2} \sigma_{X_{i}}^{2}\right) / \mu_{X_{i}}^{4}, i=1,2$ and $\stackrel{D}{\rightarrow}$ denotes convergence in distribution. Now we can estimate $\sigma_{i}^{2}$ as

$$
\hat{\sigma}_{i}^{2}=\left(\bar{X}_{i}^{2} S_{Y_{i}}^{2}+\bar{Y}_{i}^{2} S_{X_{i}}^{2}\right) / \bar{X}_{i}^{4}, \quad i=1,2
$$


where

$$
S_{x_{i}}^{2}=\frac{1}{n} \sum_{j=1}^{n}\left(X_{i j}-\bar{X}_{i}\right)^{2}, \quad S_{Y_{i}}^{2}=\frac{1}{n} \sum_{j=1}^{n}\left(Y_{i j}-Y_{i}\right)^{2}, \quad i=1,2
$$

Then $\hat{\sigma}_{i}^{2}, i=1,2$ is a strongly consistent estimator of $\sigma_{i}^{2}, i=1,2$. Again applying the Slutsky's theorem we have,

$$
\sqrt{n}\left(\begin{array}{l}
\left(\hat{\rho}_{1}-\rho_{1}\right) / \hat{\sigma}_{1} \\
\left(\hat{\rho}_{2}-\rho_{2}\right) / \hat{\sigma}_{2}
\end{array}\right) \stackrel{D}{\rightarrow} N_{2}\left(\left(\begin{array}{l}
0 \\
0
\end{array}\right),\left(\begin{array}{ll}
1 & 0 \\
0 & 1
\end{array}\right)\right)
$$

Thus $\hat{\rho}_{i}, i=1,2$ is a strongly consistent and asymptotically normal (CAN) estimator with approximate variances $\hat{\sigma}_{i}^{2} / n, i=1,2$.

\section{Consistent and Asymptotically Normal (CAN) Confidence Intervals}

Using CAN estimators $\hat{\rho}_{i}, i=1,2$ and its associated approximate variances $\hat{\sigma}_{i}^{2} / n, i=1,2$, we construct confidence intervals for intensities $\rho_{i}, i=1,2$ for a distribution-free two-stage open queueing network. Let $z_{\alpha}$ be the upper $\alpha^{t h}$ quantile of the standard normal distribution. Thus we have $100(1-\alpha) \%$ confidence intervals for $\rho_{i}, i=1,2$

$$
\left(\hat{\rho}_{i} \pm z_{a / 2} \hat{\sigma}_{i} / \sqrt{n}\right), \quad i=1,2 .
$$

\section{Variance-stabilized Bootstrap- $t$ (VST) Confidence Intervals}

According to the bootstrap procedure, a simple random sample $\left(X_{i j}^{*}, Y_{i j}^{*}, i=\right.$ $1,2, j=1,2, \cdots, n)$ called a bootstrap sample is taken from the empirical distribution function of $\left(X_{i j}, Y_{i j}, i=1,2 ; j=1,2, \cdots, n\right)$. Thus bootstrap estimate for intensity $\rho_{i}, i=1,2$ can be calculated as

$$
\hat{\rho}_{i}^{*}=\frac{\bar{y}_{i}^{*}}{\bar{x}_{i}^{*}}, i=1,2 .
$$

. The above resampling process is repeated $N$ times. The $\mathrm{N}$ bootstrap estimates $\hat{\rho}_{i 1}^{*}, \hat{\rho}_{i 2}^{*}, \cdots, \hat{\rho}_{i N}^{*}, i=1,2$ are computed from the bootstrap resample.Let $\hat{\rho}_{i}, i=$ 1,2 be strongly consistent and asymptotically normal estimator with approximate variances $\hat{\sigma}_{i}^{2} / n, i=1,2$ and consider $\hat{\sigma}_{i}=\phi\left(\hat{\rho}_{i}\right)$. To find a transformation $f\left(\hat{\rho}_{i}\right)$ such that $\operatorname{Var}\left(f\left(\hat{\rho}_{i}\right)\right) \approx$ constant,by the first order Taylor series expansion:

$f\left(\hat{\rho}_{i}\right) \approx f\left(\rho_{i}\right)+\left(\hat{\rho}_{i}-\rho_{i}\right) f^{\prime}\left(\rho_{i}\right) \Rightarrow\left[f\left(\hat{\rho}_{i}\right)-f\left(\rho_{i}\right)\right]^{2} \approx\left(\hat{\rho}_{i}-\rho_{i}\right)^{2}\left(f^{\prime}\left(\rho_{i}\right)\right)^{2}, i=1,2$.

Taking expectations on both sides, we get:

$$
\operatorname{Var}\left[f\left(\hat{\rho}_{i}\right)\right] \approx \operatorname{Var}\left(\hat{\rho}_{i}\right)\left(f^{\prime}\left(\rho_{i}\right)\right)^{2}=\left(\phi\left(\rho_{i}\right)\right)^{2}\left(f^{\prime}\left(\rho_{i}\right)\right)^{2}, \quad i=1,2 .
$$


Now consider $f\left(\hat{\rho}_{i}\right)=\sqrt{n} \log \left(\phi\left(\hat{\rho}_{i}\right)\right), i=1,2$ is the variance-stabilizing transformation. Then we have,

$$
V\left[f\left(\hat{\rho}_{i}\right)\right] \approx\left(\frac{\sqrt{n}}{\phi\left(\hat{\rho}_{i}\right)}\right)^{2} \operatorname{Var}\left[\hat{\rho}_{i}\right]=\left(\frac{\sqrt{n}}{\hat{\sigma}_{i}}\right)^{2} \operatorname{Var}\left[\hat{\rho}_{i}\right]=\frac{n}{\hat{\sigma}_{i}^{2}} \frac{\hat{\sigma}_{i}^{2}}{n}=1, i=1,2 .
$$

Here we consider $N$ bootstrap estimates $\hat{\rho}_{i 1}^{*}, \hat{\rho}_{i 2}^{*}, \cdots, \hat{\rho}_{i N}^{*}, i=1,2$ computed from the bootstrap resample. We obtain

$$
\theta_{i j}^{*}=\left(\sqrt{n} \log \left(\hat{\rho}_{i j}^{*}\right)-\sqrt{n} \log \left(\hat{\rho}_{i}\right)\right), i=1,2, j=1,2, \cdots, N .
$$

Thus we have $100(1-\alpha) \%$ Variance-stabilized Bootstrap-t(VST) confidence interval for $\rho_{i}, i=1,2$ are

$$
\left(e^{\log \left(\hat{\rho}_{i}\right)-\frac{1}{\sqrt{n}} \hat{v}_{i} t_{1-\alpha / 2}}, e^{\log \left(\hat{\rho}_{i}\right)-\frac{1}{\sqrt{n}} \hat{v}_{i} t_{\alpha / 2}}\right)
$$

where $\hat{v}_{i} t_{\alpha / 2}$ and $\hat{v}_{i} t_{1-\alpha / 2}$ are $(\alpha / 2)^{t h}$ and $(1-\alpha / 2)^{t h}$ percentile of the random sample $\theta_{i 1}^{*}, \theta_{i 2}^{*}, \cdots, \theta_{i N}^{*}, i=1,2$.

\section{Bayesian Bootstrap (BB) Confidence Intervals}

Each Bayesian Bootstrap (BB) replication generates a posterior probability for each $X_{i j}, i=1,2, j=1,2, \cdots, n$. One $\mathrm{BB}$ replication is generated by drawing $n-1$ uniform $(0,1)$ random numbers $r_{1}, r_{2}, \cdots, r_{n-1}$ ordering them, and calculating the gaps $w_{j}=r_{(j)}-r_{(j-1)}, j=1,2, \cdots, n$, where $r_{(0)}=0$ and $r_{(n)}=1$. Then $w_{i}=\left(w_{i 1}, w_{i 2}, \cdots, w_{i n}\right), i=1,2$ is the vector of probabilities attached to the inter-arrival data $X_{i j}, i=1,2, j=1,2, \cdots, n$. Considering all $\mathrm{BB}$ replications gives the $\mathrm{BB}$ distribution of the distribution of $X_{i}$ and thus of any parameter of this distribution. Hence for $\mu_{x_{i}}, i=1,2$ (the mean of $X_{i}$ ) in each $\mathrm{BB}$ replication we calculate $\mu_{x_{i}}, i=1,2$ as if $w_{i j}$ were the probability that $X_{i}=x_{i j}$ that is, we calculate $\bar{X}_{i}^{* *}=\sum_{j=1}^{n} w_{i j} x_{i j}, i=1,2$. The distribution of the values of $\bar{X}_{i}^{* *}$ over all BB replications is the BB distribution of $\mu_{X_{i}}$. Also, generating a vector of probabilities $v_{i}=\left(v_{i 1}, v_{i 2}, \cdots, v_{i n}\right), i=1,2$ attached to the service time data values $Y_{i j}, i=1,2, j=1,2, \cdots, n$ in a BB replication, and we calculate $\bar{Y}_{i}^{* *}=\sum_{j=1}^{n} v_{i j} y_{i j}$ for $\mu_{Y_{j}}$ (the mean of $Y_{i}$ ). An estimate of intensity $\rho_{i}$ can be calculated from BB replications as $\hat{\rho}_{i}^{* *}=\frac{\bar{Y}_{i}^{* *}}{\bar{X}_{i}^{* *}}, i=1,2$, where $\hat{\rho}_{i}^{* *}, i=1,2$ is called a Bayesian bootstrap estimate of $\rho_{i}, i=1,2$. The above BB process can be repeated $N$ times. The $N B B$ estimates $\hat{\rho}_{i 1}^{*}, \hat{\rho}_{i 2}^{*}, \cdots, \hat{\rho}_{i N}^{*}, i=1,2$ can be computed from the $B B$ replications. Averaging the $N B B$ estimates, we obtain 
that $\hat{\rho}_{B B}^{\prime}(i)=\frac{1}{N} \sum_{j=1}^{N} \hat{\rho}_{i j}^{* *}, i=1,2$ is the $B B$ estimate of $\rho_{i}, i=1,2$. Also the standard deviation of $\hat{\rho}_{i}$ can be estimated by

$$
s d\left(\hat{\rho}_{B B}^{\prime}(i)\right)=\left\{\frac{1}{N-1} \sum_{j=1}^{N}\left(\hat{\rho}_{i j}^{* *}-\hat{\rho}_{B B}^{\prime}(i)\right)^{2}\right\}^{1 / 2}, i=1,2 .
$$

Applying the asymptotic normality of $\hat{\rho}_{i}, i=1,2$, we have $100(1-\alpha) \% B B$ confidence interval for $\rho_{i}, i=1,2$ are

$$
\left(\hat{\rho}_{i} \pm z_{\alpha / 2} s d\left(\hat{\rho}_{B B}^{\prime}(i)\right)\right), \quad i=1,2 .
$$

\section{Percentile Bootstrap (PB) Confidence Intervals}

Now call $\hat{\rho}_{i 1}^{*}, \hat{\rho}_{i 2}^{*}, \cdots, \hat{\rho}_{i N}^{*}, i=1,2$ the bootstrap distribution of $\hat{\rho}_{i}, i=1,2$. Let $\hat{\rho}_{i}^{*}(1), \hat{\rho}_{i}^{*}(2), \cdots, \hat{\rho}_{i}^{*}(N), i=1,2$ be the order statistics of $\hat{\rho}_{i 1}^{*}, \hat{\rho}_{i 2}^{*}, \cdots, \hat{\rho}_{i N}^{*}, i=$ 1,2 . Then utilizing the $100(\alpha / 2)^{t h}$ and $100(1-\alpha / 2)^{t h}$ percentage points of the bootstrap distribution, $100(1-\alpha) \%$ PB confidence interval for $\rho_{i}, i=1,2$ are obtained as

$$
\left(\hat{\rho}_{i}^{*}\left(\left[N\left(\frac{\alpha}{2}\right)\right]\right), \hat{\rho}_{i}^{*}\left(\left[N\left(1-\frac{\alpha}{2}\right)\right]\right)\right), i=1,2
$$

where $[x]$ denotes the greatest integer less than or equal to $x$.

\section{Simulation Study}

A numerical simulation study was undertaken to evaluate performance of the various interval estimation approaches mentioned above for a two-stage open queueing network with short run. It is observed that most statisticians assess performances of interval estimations in terms of coverage percentages or average lengths of confidence intervals. However, through simulation study in the research work, we find that larger coverage percentages of confidence interval may often be due to wider standard deviation of interval estimation methods. Moreover, narrower confidence intervals may often lead to smaller coverage percentages. Hence, both coverage percentage and average length are not efficient for appraising interval estimation methods. In order to overcome above two shortcomings, we consider a measure, named relative coverage, to evaluate performances of interval estimation methods. 
Table-1 : Different levels of intensity parameters considered in the simulation study

\begin{tabular}{|l|l|}
\hline \multicolumn{1}{|c|}{$\rho_{1}<\rho_{2}$} & \multicolumn{1}{c|}{$\rho_{1}>\rho_{2}$} \\
\hline (1) Low=0.1 and Moderate=0.5 & (1) Moderate=0.5 and Low=0.1 \\
(2) Low=0.1 and High=0.9 & (2) High=0.9 and Low=0.1 \\
(3) Moderate=0.5 and High=0.9 & (3) High=0.9 and Moderate=0.5 \\
\hline
\end{tabular}

Relative coverage is defined as the ratio of coverage percentage to average length of confidence interval. Larger relative coverage implies the better performances of the corresponding confidence intervals. In order to reach this goal, we not only set a continuous distribution with mean $1 / \lambda$ on inter-arrival time $X_{1}$ and $X_{2}$ but also assume a continuous distribution with mean $1 / \mu_{1}$ on the service time $Y_{1}$ at node- 1 and that of $1 / \mu_{2}$ on $Y_{2}$ at node-2.

Table-2 : Different queueing network models simulated for study

\begin{tabular}{|c|c|c|c|c|c|}
\hline $\begin{array}{c}\text { Queueing } \\
\text { Networks } \\
\text { type }\end{array}$ & $\begin{array}{c}\text { Models } \\
\text { simulated }\end{array}$ & $\begin{array}{c}\text { C.V. of } \\
\text { inter-arrival } \\
\text { time for node-1 }\end{array}$ & $\begin{array}{c}\text { C.V. of } \\
\text { inter- arrival } \\
\text { time for node-2 }\end{array}$ & $\begin{array}{c}\text { C.V. of } \\
\text { service times } \\
\text { for node-1 }\end{array}$ & $\begin{array}{c}\text { C.V. of } \\
\text { forvice node-2 }\end{array}$ \\
\hline $\begin{array}{c}\text { M/G/1 to } \\
\text { G/M/1 }\end{array}$ & $M / E_{4} / 1$ to $E_{4} / M / 1$ & 1 & $1 / 2$ & $1 / 2$ & 1 \\
\cline { 2 - 6 } & $M / H_{4}^{P e} / 1$ to $H_{4}^{P e} / M / 1$ & 1 & $>1$ & $>1$ & 1 \\
\hline G/G/1 to & $E_{4} / H_{4}^{P e} / 1$ to $H_{4}^{P e} / E_{4} / 1$ & $1 / 2$ & $>1$ & $>1$ & $1 / 2$ \\
\cline { 2 - 6 } G/G/1 & $E_{4} / H_{4}^{P o} / 1$ to $H_{4}^{P o} / E_{4} / 1$ & $1 / 2$ & $<1$ & $<1$ & $1 / 2$ \\
\hline
\end{tabular}

For each level of $\rho_{i}, i=1,2$ random samples of inter-arrival times and service times $\left(X_{i j}, Y_{i j}, i=1,2, j=1,2, \cdots, n\right.$ are drawn from $\left(X_{i}, Y_{i}, i=1,2\right)$. Next $N=1000$ bootstrap resamples each of size $n=10,20 \& 25$ are drawn from the original samples, as well as $\mathrm{N}=1000 \mathrm{BB}$ replications are simulated for the original samples. According to equations (4) to (8) we obtain CAN, VST, BB and PB confidence intervals of intensities $\rho_{1}$ and $\rho_{2}$ with confidence level $90 \%$. The above simulation process is replicated $N=1000$ times and we compute coverage percentages, average lengths and relative coverage of the above mentioned confidence intervals. We utilize a PC Dual Core and apply Matlab 7.0.1 to accomplish all simulations. Here C.V. represents coefficient of variation corresponding to the inter-arrival/service time distribution, $\mathrm{M}$ represents an exponential distribution, $E_{4}$ a 4-stage Erlang distribution, $H_{4}^{P e}$ a 4-stage hyperexponential distribution and $H_{4}^{P o}$ a 4-stage hypo-exponential distribution. 
Table-7 : Performances of the estimation approaches of intensities $\rho_{1}$ and $\rho_{2}$ under various Queueing Networks

\begin{tabular}{|c|c|c|c|c|c|c|}
\hline $\begin{array}{c}\text { Queueing } \\
\text { Network } \\
\text { Type }\end{array}$ & $\begin{array}{l}\text { Queuing Network } \\
\text { simulated }\end{array}$ & $\begin{array}{l}\text { Queueing Network } \\
\text { with greater } \\
\text { relative coverage }\end{array}$ & $\begin{array}{c}\text { Intensity } \\
\text { Parameters }\end{array}$ & $\begin{array}{r}\begin{array}{r}\text { Estin } \\
\\
\text { rel }\end{array} \\
n=1\end{array}$ & $\begin{array}{l}\text { lation a } \\
\text { ith gre } \\
\text { tive co } \\
n=2\end{array}$ & $\begin{array}{l}\text { proach } \\
\text { est } \\
\text { erage } \\
n=25\end{array}$ \\
\hline & & & $\begin{array}{c}\rho_{1}=0.1 \\
\& \rho_{2}=0.5\end{array}$ & $\begin{array}{l}\text { VST } \\
\text { BB }\end{array}$ & $\begin{array}{c}\text { CAN } \\
\text { BB }\end{array}$ & $\begin{array}{c}\text { CAN } \\
\text { BB }\end{array}$ \\
\hline & & & $\rho_{1}=0.1$ & CAN & CAN & VST \\
\hline & & & $\& \rho_{2}=0.9$ & $\mathrm{BB}$ & $\mathrm{BB}$ & $\mathrm{BB}$ \\
\hline$M / G / 1$ & $M / E_{4} / 1$ to $E_{4} / M / 1$ & $M / E_{4} / 1$ & $\begin{array}{c}\rho_{1}=0.5 \\
\& \rho_{2}=0.1\end{array}$ & $\begin{array}{l}\text { VST } \\
\text { BB }\end{array}$ & $\begin{array}{c}\text { CAN } \\
\text { BB }\end{array}$ & $\begin{array}{l}\text { VST } \\
\text { BB }\end{array}$ \\
\hline $\begin{array}{c}\text { to } \\
G / M / 1\end{array}$ & and & $\begin{array}{c}\text { to } \\
E_{4} / M / 1\end{array}$ & $\begin{array}{c}\rho_{1}=0.5 \\
\& \rho_{2}=0.9\end{array}$ & $\begin{array}{l}\text { VST } \\
\text { BB }\end{array}$ & $\begin{array}{c}\text { CAN } \\
\text { BB }\end{array}$ & $\begin{array}{l}\text { VST } \\
\text { PB }\end{array}$ \\
\hline & $/ M / 1$ & & $\rho_{1}=0.9$ & CAN & VST & VST \\
\hline & & & $\& \rho_{2}=0.1$ & $\mathrm{BB}$ & PB & $\mathrm{BB}$ \\
\hline & & & $\rho_{1}=0.9$ & VST & VST & $\mathrm{BB}$ \\
\hline & & & $\& \rho_{2}=0.5$ & $\mathrm{BB}$ & $\mathrm{BB}$ & PB \\
\hline \multirow{12}{*}{$\begin{array}{l}G / G / 1 \\
\text { to } \\
G / G / 1\end{array}$} & \multirow{8}{*}{$E_{4} / H_{4}^{p e} / 1$ to $H_{4}^{P e} / E_{4} / 1$} & \multirow{12}{*}{$\begin{array}{c}E_{4} / H_{4}^{P e} / 1 \\
\text { to } \\
H_{4}^{P e} / E_{4} / 1\end{array}$} & $\rho_{1}=0.1$ & $\mathrm{BB}$ & $\mathrm{BB}$ & $\mathrm{BB}$ \\
\hline & & & $\& \rho_{2}=0.5$ & $\mathrm{BB}$ & $\mathrm{BB}$ & $\mathrm{BB}$ \\
\hline & & & $\rho_{1}=0.1$ & $\overline{\mathrm{BB}}$ & $\overline{\mathrm{BB}}$ & $\overline{\mathrm{BB}}$ \\
\hline & & & $\& \rho_{2}=0.9$ & $\mathrm{BB}$ & $\mathrm{BB}$ & $\mathrm{BB}$ \\
\hline & & & $\rho_{1}=0.5$ & $\mathrm{BB}$ & $\mathrm{BB}$ & $\mathrm{BB}$ \\
\hline & & & $\& \rho_{2}=0.1$ & $\mathrm{BB}$ & $\mathrm{BB}$ & $\mathrm{BB}$ \\
\hline & & & $\rho_{1}=0.5$ & $\mathrm{BB}$ & $\mathrm{BB}$ & $\mathrm{BB}$ \\
\hline & & & $\& \rho_{2}=0.9$ & $\mathrm{BB}$ & $\mathrm{BB}$ & $\mathrm{BB}$ \\
\hline & \multirow[t]{4}{*}{$E_{4} / H_{4}^{P o} / 1$ to $H_{4}^{P o} / E_{4} / 1$} & & $\rho_{1}=0.9$ & $\mathrm{BB}$ & $\mathrm{BB}$ & $\mathrm{BB}$ \\
\hline & & & $\& \rho_{2}=0.1$ & $\mathrm{BB}$ & $\mathrm{BB}$ & $\mathrm{BB}$ \\
\hline & & & $\rho_{1}=0.9$ & $\mathrm{BB}$ & $\mathrm{BB}$ & $\mathrm{BB}$ \\
\hline & & & $\& \rho_{2}=0.5$ & $\mathrm{BB}$ & $\mathrm{BB}$ & $\mathrm{BB}$ \\
\hline
\end{tabular}

Based on the above mentioned interval estimation approaches the coverage percentage, average lengths and relative coverage of intensities $\rho_{1}$ and $\rho_{2}$ are shown in Tables 3 to 6 (see Appendix) for queuing network models (presented in Table 2) with short run. According to the simulation results in Tables 3 to 6, we find that average lengths are decreases but both coverage percentages and relative coverage are increases with sample size $n$. Also we observe that the coverage percentage can approaches to $90 \%$ when $\mathrm{n}$ increases to 25 .

Based on Table 7, we note that:

(1) Under $M / G / 1$ to $G / M / 1$ model the confidence intervals corresponding to queueing network with inter-arrival distribution and service time distribution of small $\mathrm{CV}(<1)$ have greater relative coverage than those of large $\mathrm{CV}(>1)$ for intensities $\rho_{1}$ and $\rho_{2}$. The estimation approaches Consistent and Asymptotically Normal estimator, Variancestabilized Bootstrap-t and Bayesian bootstrap has the greatest relative 
coverage. Also the confidence intervals of $M / E_{4} / 1$ to $E_{4} / M / 1$ shows the greatest relative coverage for $\rho_{1}$ and $\rho_{2}$.

(2) Under $G / G / 1$ to $G / G / 1$ models the confidence interval corresponding to queueing network models with inter-arrival distribution and service time distribution of large $\mathrm{CV}(>1)$ have greatest relative coverage than those of small $\mathrm{CV}(<1)$ for intensities $\rho_{1}$ and $\rho_{2}$. The estimation approach Bayesian bootstrap has the greatest relative coverage. Also the confidence intervals of $E_{4} / H_{4}^{P e} / 1$ to $H_{4}^{P e} / E_{4} / 1$ shows the greatest relative coverage for $\rho_{1}$ and $\rho_{2}$.

(3) Average lengths are decreases and relative coverage increases with $\mathrm{n}$ increases for $\rho_{1}$ and $\rho_{2}$.

Based upon our additional simulation study (not be presented), all the above mentioned approaches perform almost equally well on the interval estimation for intensities $\rho_{1}$ and $\rho_{2}$ when the sample size $\mathrm{n}$ is sufficiently large.

\section{Conclusions}

This paper provides the interval estimations of intensities $\rho_{1}$ and $\rho_{2}$ for twostage open queueing network with short run data. Different estimation approaches CAN, VST, BB and PB are applied to produce confidence intervals for intensities $\rho_{1}$ and $\rho_{2}$. The relative coverage is adopted to understand, compare and assess performance of the resulted confidence intervals. The simulation results imply that the CAN, VST and BB method has the best performance for $M / G / 1$ to $G / M / 1$ queueing network and under $G / G / 1$ to $G / G / 1$ queueing networks, the estimation approach BB out performs. The above mentioned approaches are easily applied to practical queueing network such as all types of open, closed, mixed queueing networks as well as cyclic, retrial queueing models. Further research may consider investigations of other characteristics of a queueing network with small sample data by using the different estimation approaches. 


\section{Appendix:}

Table-3 : Simulation results of coverage percentage, average lengths and relative coverage for $90 \%$ confidence intervals of queueing Network $M / E_{4} / 1$ to $E_{4} / M / 1$

\begin{tabular}{|c|c|c|c|c|c|c|c|c|c|c|}
\hline \multirow{2}{*}{$\begin{array}{c}\text { Intensity } \\
\text { Parameters }\end{array}$} & \multirow{2}{*}{$\begin{array}{l}\text { Estimation } \\
\text { Approches }\end{array}$} & \multicolumn{3}{|c|}{ Coverage Percentages } & \multicolumn{3}{|c|}{ Average Lengths } & \multicolumn{3}{|c|}{ Relative Coverage } \\
\hline & & $n=1$ & $n=20$ & $n=2$ & $n=10$ & $n=2$ & $n=25$ & $n=10$ & $n=20$ & $n=25$ \\
\hline \multirow{8}{*}{$\begin{array}{c}\rho_{1}=0.1 \\
\& \\
\rho_{2}=0.5\end{array}$} & CAN1 & 0.857 & 0.873 & 0.900 & 0.121 & 0.084 & 0.075 & 7.095 & 10.346 & 12.057 \\
\hline & CAN2 & 0.859 & 0.879 & 0.889 & 0.569 & 0.402 & 0.368 & 1.509 & 2.186 & 2.415 \\
\hline & VST1 & 0.838 & 0.856 & 0.886 & 0.118 & 0.083 & 0.074 & 7.125 & 10.297 & 12.009 \\
\hline & VST2 & 0.879 & 0.878 & 0.879 & 0.649 & 0.425 & 0.383 & 1.354 & 2.065 & 2.295 \\
\hline & $\mathrm{BB} 1$ & 0.859 & 0.873 & 0.897 & 0.125 & 0.085 & 0.075 & 6.893 & 10.286 & 12.008 \\
\hline & BB2 & 0.837 & 0.867 & 0.881 & 0.532 & 0.389 & 0.358 & 1.574 & 2.231 & 2.463 \\
\hline & PB1 & 0.828 & 0.855 & 0.872 & 0.136 & 0.089 & 0.078 & 6.074 & 9.606 & 11.247 \\
\hline & PB2 & 0.860 & 0.880 & 0.886 & 0.552 & 0.395 & 0.362 & 1.559 & 2.226 & 2.447 \\
\hline \multirow{8}{*}{$\begin{array}{c}\rho_{1}=0.1 \\
\& \\
\rho_{2}=0.9\end{array}$} & CAN1 & 0.861 & 0.898 & 0.880 & 0.121 & 0.084 & 0.074 & 7.131 & 10.749 & 11.911 \\
\hline & CAN2 & 0.835 & 0.853 & 0.876 & 1.008 & 0.714 & 0.652 & 0.829 & 1.195 & 1.343 \\
\hline & VST1 & 0.831 & 0.882 & 0.877 & 0.117 & 0.082 & 0.073 & 7.087 & 10.714 & 11.999 \\
\hline & VST2 & 0.861 & 0.862 & 0.868 & 1.154 & 0.754 & 0.681 & 0.746 & 1.143 & 1.274 \\
\hline & BB1 & 0.867 & 0.898 & 0.874 & 0.125 & 0.084 & 0.074 & 6.950 & 10.688 & 11.827 \\
\hline & BB2 & 0.817 & 0.848 & 0.869 & 0.939 & 0.690 & 0.634 & 0.870 & 1.229 & 1.371 \\
\hline & PB1 & 0.830 & 0.869 & 0.865 & 0.136 & 0.088 & 0.077 & 6.090 & 9.868 & 11.254 \\
\hline & PB2 & 0.834 & 0.854 & 0.868 & 0.977 & 0.702 & 0.643 & 0.853 & 1.217 & 1.349 \\
\hline \multirow{8}{*}{$\begin{array}{c}\rho_{1}=0.5 \\
\& \\
\rho_{2}=0.1\end{array}$} & CAN1 & 0.867 & 0.900 & 0.888 & 0.597 & 0.417 & 0.374 & 1.452 & 2.158 & 2.377 \\
\hline & CAN2 & 0.864 & 0.860 & 0.857 & 0.112 & 0.081 & 0.072 & 7.713 & 10.604 & 11.837 \\
\hline & VST1 & 0.852 & 0.874 & 0.882 & 0.581 & 0.410 & 0.370 & 1.466 & 2.129 & 2.386 \\
\hline & VST2 & 0.8 & 0.857 & 0.870 & 0.127 & 0.085 & 0.075 & 6.866 & 10.043 & 11.546 \\
\hline & BB1 & 0.868 & 0.900 & 0.887 & 0.616 & 0.420 & 0.3 & 1.408 & 2.143 & 2.372 \\
\hline & BB2 & 0 . & 0.852 & 0.849 & 0.104 & 0.078 & 0.070 & 8.061 & 10.866 & 12.076 \\
\hline & $\mathrm{Pl}$ & 0.831 & 0.865 & 0.885 & 0.675 & 0.439 & 0. & 1.231 & 1.973 & 2.277 \\
\hline & 2 & 0.856 & 0.854 & 0.861 & 0.109 & 0.080 & 0.071 & 7.873 & 10.726 & 12.074 \\
\hline \multirow{8}{*}{$\begin{array}{c}\rho_{1}=0.5 \\
\& \\
\rho_{2}=0.9\end{array}$} & N1 & 0.852 & 0.886 & 0.877 & 0.589 & 0.419 & 0.376 & 1.446 & 2.116 & 2.332 \\
\hline & N2 & 0.836 & 0.869 & 0.875 & 1.020 & 0.726 & 0.641 & 0.819 & 1.197 & 1.366 \\
\hline & VST1 & 0.841 & 0.873 & 0.866 & 0.573 & 0.413 & 0.371 & 1.466 & 2.115 & 2.333 \\
\hline & VST2 & 0.832 & 0.875 & 0.883 & 1.152 & 0.769 & 0.669 & 0.722 & 1.138 & 1.321 \\
\hline & BB1 & 0.853 & 0.880 & 0.874 & 0.606 & 0.421 & 0.377 & 1.408 & 2.092 & 2.321 \\
\hline & BB2 & 0.813 & 0.858 & 0.870 & 0.953 & 0.701 & 0.624 & 0.854 & 1.225 & 1.395 \\
\hline & PB1 & 0.836 & 0.866 & 0.869 & 0.660 & 0.439 & 0.391 & 1.267 & 1.973 & 2.224 \\
\hline & PB2 & 0.817 & 0.868 & 0.885 & 0.990 & 0.715 & 0.631 & 0.825 & 1.215 & 1.402 \\
\hline \multirow{8}{*}{$\begin{array}{c}\rho_{1}=0.9 \\
\& \\
\rho_{2}=0.1\end{array}$} & $\mathrm{C}$ & 0.855 & 0.878 & 0.8 & 1.112 & 0.756 & 0.666 & 0.769 & 1.161 & 1.311 \\
\hline & & 42 & 0.873 & 0. & 0.1 & 0.081 & & 7.488 & 10.767 & 11.967 \\
\hline & & 0.832 & 0.870 & 0.867 & 1.0 & 0.744 & & 0.768 & 1.170 & 1.318 \\
\hline & VST2 & 0.837 & 0.876 & 0.875 & 0.127 & 0.086 & 0.076 & 6.604 & 10.236 & 11.531 \\
\hline & BB1 & 0.861 & 0.877 & 0.872 & 1.1 & 0.759 & 0.666 & 0.753 & 1.155 & 1.309 \\
\hline & BB2 & 0 . & 0.861 & 0.860 & 0.105 & 0.078 & 0.071 & 7.805 & 10.997 & 12.175 \\
\hline & PB 1 & 0.819 & 0.842 & 0.869 & 1.249 & 0.797 & 0.692 & 0.656 & 1.057 & 1.255 \\
\hline & PB2 & 0.836 & 0.877 & 0.865 & 0.109 & 0.080 & 0.072 & 7.656 & 11.030 & 12.090 \\
\hline \multirow{8}{*}{$\begin{array}{c}\rho_{1}=0.9 \\
\& \\
\rho_{2}=0.5\end{array}$} & CAN1 & 0.879 & 0.874 & 0.887 & 1.109 & 0.751 & 0.676 & 0.793 & 1.164 & 1.312 \\
\hline & CAN2 & 0.840 & 0.864 & 0.860 & 0.550 & 0.406 & 0.356 & 1.527 & 2.130 & 2.419 \\
\hline & $\Gamma 1$ & 0.871 & 0.876 & 0.870 & 1.079 & 0.740 & 0.667 & 0.807 & 1.184 & 1.304 \\
\hline & VST2 & 0.857 & 0.862 & 0.875 & 0.620 & 0.428 & 0.371 & 1.382 & 2.012 & 2.357 \\
\hline & BB1 & 0.883 & 0.883 & 0.888 & 1.147 & 0.754 & 0.675 & 0.770 & 1.171 & 1.315 \\
\hline & BB2 & & 0.856 & 0.852 & 0.513 & 0.391 & 0.346 & 1.598 & 2.187 & 2.462 \\
\hline & PB1 & 0.859 & 0.875 & 0.862 & 1.259 & 0.792 & 0.703 & 0.682 & 1.105 & 1.227 \\
\hline & PB2 & 0.840 & 0.865 & 0.867 & 0.533 & 0.398 & 0.351 & 1.576 & 2.173 & 2.472 \\
\hline
\end{tabular}

Stat., Optim. Inf. Comput. Vol. 2, March 2014. 
Table-4 : Simulation results of coverage percentage, average lengths and relative coverage for $90 \%$ confidence intervals of queueing Network $M / H_{4}^{P e} / 1$ to

$$
H_{4}^{P e} / M / 1
$$

\begin{tabular}{|c|c|c|c|c|c|c|c|c|c|c|}
\hline \multirow{2}{*}{$\begin{array}{c}\text { Intensity } \\
\text { Parameters }\end{array}$} & \multirow{2}{*}{$\begin{array}{l}\text { Estimation } \\
\text { Approches }\end{array}$} & \multicolumn{3}{|c|}{ Coverage Percentages } & \multicolumn{3}{|c|}{ Average Lengths } & \multicolumn{3}{|c|}{ Relative Coverage } \\
\hline & & \multicolumn{9}{|c|}{$n=10|n=20| n=25|n=10| n=20|n=25| n=10|n=20| n=2$} \\
\hline \multirow{8}{*}{$\begin{array}{c}\rho_{1}=0.1 \\
\& \\
\rho_{1}=0.5\end{array}$} & CAN1 & 0.884 & 0.897 & 0.892 & 0.128 & 0.088 & 0.077 & 6.916 & 10.246 & 11.615 \\
\hline & CAN2 & 0.855 & 0.873 & 0.882 & 0.578 & 0.420 & 0.379 & 1.478 & 2.078 & 2.325 \\
\hline & VST1 & 0.846 & 0.869 & 0.891 & 0.126 & 0.087 & 0.076 & 6.727 & 10.007 & 11.678 \\
\hline & VST2 & 0.866 & 0.872 & 0.886 & 0.648 & 0.441 & 0.394 & 1.336 & 1.975 & 2.248 \\
\hline & BB 1 & 0.886 & 0.899 & 0.891 & 0.131 & 0.088 & 0.077 & 6.773 & 10.233 & 11.597 \\
\hline & BB2 & 0.836 & 0.861 & 0.877 & 0.543 & 0.407 & 0.370 & 1.539 & 2.117 & 2.370 \\
\hline & PB 1 & 0.833 & 0.860 & 0.886 & 0.141 & 0.092 & 0.080 & 5.892 & 9.375 & 11.113 \\
\hline & PB2 & 0.866 & 0.865 & 0.878 & 0.568 & 0.414 & 0.375 & 1.525 & 2.088 & 2.343 \\
\hline \multirow{8}{*}{$\begin{array}{c}\rho_{1}=0.1 \\
\& \\
\rho_{1}=0.9\end{array}$} & CAN1 & 0.870 & 0.881 & 0.869 & .126 & 0.086 & 0.078 & 6.919 & 10.205 & 11.198 \\
\hline & $\mathrm{AN} 2$ & 0.852 & 0.854 & 0.870 & .063 & 0.744 & 0.676 & 0.802 & 1.148 & 1.286 \\
\hline & VST1 & 0.848 & 0.879 & 0.860 & 0.124 & 0.086 & 0.077 & 6.853 & 10.252 & 11.162 \\
\hline & VST2 & 0.853 & 0.854 & 0.876 & 1.201 & 0.782 & 0.703 & 0.710 & 1.092 & 1.245 \\
\hline & BB1 & 0.868 & 0.883 & 0.867 & 0.130 & 0.087 & 0.078 & 6.692 & 10.172 & 11.159 \\
\hline & BB2 & 0.831 & 0.847 & 0.862 & 0.999 & 0.721 & 0.660 & 0.832 & 1.174 & 1.306 \\
\hline & PB1 & 0.841 & 0.876 & 0.850 & 0.141 & 0.090 & 0.080 & 5.982 & 9.682 & 10.575 \\
\hline & PB2 & 0.842 & 0.850 & 0.871 & 1.041 & 0.735 & 0.669 & 0.809 & 1.156 & 1.302 \\
\hline \multirow{8}{*}{$\begin{array}{c}\rho_{1}=0.5 \\
\& \\
\rho_{1}=0.1\end{array}$} & CAN1 & 0.871 & 0.890 & & 40 & 37 & 86 & 1.360 & 2.036 & 2.316 \\
\hline & CAN2 & 45 & 0.875 & 0.867 & 18 & 0.085 & 0.075 & 7.154 & 10.310 & 11.537 \\
\hline & T1 & 47 & 0.874 & & 30 & 0. & 83 & 44 & 2.019 & 2.316 \\
\hline & VST2 & 0 & 0.864 & 0.877 & 33 & 0.089 & 0.078 & 38 & 9.676 & 11.210 \\
\hline & & 0 & 0.890 & & 58 & 39 & & 40 & 2.027 & 2.305 \\
\hline & B & 20 & 0.856 & & 11 & 82 & & 81 & 10.418 & 11.649 \\
\hline & & 32 & 0.867 & & 20 & & & & 1.892 & 2.233 \\
\hline & PB2 & 0.838 & 0.858 & 0.876 & 16 & 0.084 & 0.074 & 7.244 & 10.241 & 11.787 \\
\hline \multirow{8}{*}{$\begin{array}{c}\rho_{1}=0.5 \\
\& \\
\rho_{1}=0.9\end{array}$} & AN1 & 0.857 & 0.886 & 0.890 & 641 & 0.436 & 89 & 1.337 & 2.034 & 2.287 \\
\hline & $\mathrm{N} 2$ & 0.811 & 0.871 & 0.858 & 028 & 0.752 & 0.681 & 0.789 & 1.159 & 1.261 \\
\hline & VST1 & 0.825 & 0.876 & 0.883 & 0.632 & 0.431 & 0.387 & 1.306 & 2.031 & 2.284 \\
\hline & VST2 & 0.830 & 0.875 & 0.859 & 1.148 & 0.790 & 0.708 & 0.723 & 1.108 & 1.214 \\
\hline & BB 1 & 0.857 & 0.886 & 0.891 & 0.666 & 0.437 & 0.391 & 1.287 & 2.027 & 2.279 \\
\hline & BB2 & 0.788 & 0.861 & 0.851 & 0.967 & 0.729 & 0.663 & 0.815 & 1.181 & 1.283 \\
\hline & PB 1 & 0.813 & 0.865 & 0.885 & 0.719 & 0.457 & 0.404 & 1.130 & 1.894 & 2.191 \\
\hline & & 22 & 0.873 & 0.8 & 1.007 & 0.742 & 0.674 & 0.817 & 1.176 & 1.268 \\
\hline \multirow{8}{*}{$\begin{array}{c}\rho_{1}=0.9 \\
\& \\
\rho_{1}=0.1\end{array}$} & & 61 & 0.882 & & & 80 & 00 & 48 & 1.131 & 1.269 \\
\hline & & & 0 & & 6 & 83 & 74 & 31 & 10.650 & 11.891 \\
\hline & & & 0.865 & & & & & & 1.116 & 1.272 \\
\hline & VST2 & 347 & 0.877 & 0.886 & 30 & 0.088 & 0.077 & 6.492 & 9.969 & 11.458 \\
\hline & & 61 & 0.881 & & & & & 0.726 & 1.123 & 1.269 \\
\hline & BB2 & 0.814 & 0.877 & 0.874 & 0.109 & 0.081 & 0.073 & 7.462 & 10.841 & 12.054 \\
\hline & & 0.834 & 0.862 & 0.876 & 1.292 & 0.820 & 0.727 & 0.646 & 1.051 & 1.205 \\
\hline & PB2 & 0.832 & 0.872 & 0.878 & 0.114 & 0.082 & 0.074 & 7.294 & 10.578 & 11.942 \\
\hline \multirow{8}{*}{$\begin{array}{c}\rho_{1}=0.9 \\
\& \\
\rho_{1}=0.5\end{array}$} & CAN1 & 0.872 & 0.886 & 0.889 & 1.128 & 0.789 & 0.702 & 0.773 & 1.123 & 1.267 \\
\hline & $\mathrm{N} 2$ & 0.868 & 0.863 & 0.874 & 0.591 & 0.426 & 0.383 & 1.470 & 2.024 & 2.285 \\
\hline & & 0.848 & 0.882 & 0.870 & 1.111 & 0.783 & 0.695 & 0.763 & 1.126 & 1.251 \\
\hline & VST2 & 0.853 & 0.871 & 0.891 & 0.664 & 0.450 & 0.398 & 1.285 & 1.936 & 2.237 \\
\hline & & & & & & 0.796 & 0.703 & 0.749 & 1.111 & 1.261 \\
\hline & BB2 & 0.847 & 0.854 & 0.862 & 0.555 & 0.414 & 0.373 & 1.526 & 2.063 & 2.314 \\
\hline & PB 1 & 0.825 & 0.879 & & 1.272 & 0.829 & & 0.649 & 1.061 & 1.185 \\
\hline & PB2 & 0.858 & 0.860 & 0.883 & 0.579 & 0.421 & 0.379 & 1.482 & 2.041 & 2.332 \\
\hline
\end{tabular}

Stat., Optim. Inf. Comput. Vol. 2, March 2014. 
Table-5 : Simulation results of coverage percentage, average lengths and relative coverage for $90 \%$ confidence intervals of queueing Network $E_{4} / H_{4}^{P e} / 1$ to

$$
H_{4}^{P e} / E_{4} / 1
$$

\begin{tabular}{|c|c|c|c|c|c|c|c|c|c|c|}
\hline \multirow{2}{*}{$\begin{array}{c}\text { Intensity } \\
\text { Parameters }\end{array}$} & \multirow{2}{*}{$\begin{array}{l}\text { Estimation } \\
\text { Approches }\end{array}$} & \multicolumn{3}{|c|}{ Coverage Percentages } & \multicolumn{3}{|c|}{ Average Lengths } & \multicolumn{3}{|c|}{ Relative Coverage } \\
\hline & & \multicolumn{9}{|c|}{$n=10|n=20| n=25 n=10|n=20| n=25 \mid n=10 n n=20 n=25$} \\
\hline \multirow{8}{*}{$\begin{array}{c}\rho_{1}=0.1 \\
\& \\
\rho_{1}=0.5\end{array}$} & CAN1 & 0.853 & 0.878 & 0.890 & 0.080 & 0.057 & 0.051 & 10.605 & 15.531 & 17.598 \\
\hline & CAN2 & 0.865 & 0.881 & 0.900 & 0.407 & 0.284 & 0.252 & 2.124 & 3.107 & 3.570 \\
\hline & VST1 & 0.843 & 0.885 & 0.889 & 0.080 & 0.056 & 0.050 & 10.506 & 15.709 & 17.625 \\
\hline & VST2 & 0.826 & 0.860 & 0.899 & 0.399 & 0.280 & 0.249 & 2.071 & 3.070 & 3.608 \\
\hline & BB1 & 0.830 & 0.866 & 0.880 & 0.075 & 0.055 & 0.049 & 11.032 & 15.859 & 17.924 \\
\hline & BB2 & 0.846 & 0.866 & 0.897 & 0.383 & 0.274 & 0.245 & 2.207 & 3.157 & 3.664 \\
\hline & & 0.836 & 0.878 & 0.892 & 0.079 & 0.056 & 0.050 & 10.624 & 15.758 & 17.833 \\
\hline & & 0.839 & 0.870 & 0.896 & 0.404 & 0.281 & 0.250 & 2.077 & 3.094 & 3.585 \\
\hline \multirow{8}{*}{$\begin{array}{c}\rho_{1}=0.1 \\
\& \\
\rho_{1}=0.9\end{array}$} & & 0.888 & 0.876 & 0.888 & 0.081 & 0.057 & 0.051 & 10.997 & 15.345 & 17.530 \\
\hline & & 0.872 & 0.887 & 0.890 & 0.730 & 0.513 & 0.460 & 1.195 & 1.729 & 1.934 \\
\hline & VST1 & 0.867 & 0.866 & 0.883 & 0.081 & 0.057 & 0.050 & 10.745 & 15.222 & 17.498 \\
\hline & VST2 & 0.856 & 0.884 & 0.878 & 0.715 & 0.508 & 0.457 & 1.198 & 1.740 & 1.923 \\
\hline & BB1 & 0.867 & 0.860 & 0.875 & 0.075 & 0.055 & 0.049 & 11.504 & 15.602 & 17.808 \\
\hline & B & 0.854 & 0.874 & 0.881 & 0.688 & 0.495 & 0.447 & 1.241 & 1.767 & 1.972 \\
\hline & & & 0.865 & 0.882 & 0.079 & 0.056 & 0.050 & 11.085 & 15.348 & 17.635 \\
\hline & $\mathrm{P}$ & 0.858 & 0.884 & 0.8 & 0.726 & 0.510 & 0.457 & 1.181 & 1.733 & 1.905 \\
\hline \multirow{8}{*}{$\begin{array}{c}\rho_{1}=0.5 \\
\& \\
\rho_{1}=0.1\end{array}$} & & 71 & 0.871 & & 99 & .282 & 55 & 2.184 & 3.093 & 3.484 \\
\hline & & & 0.897 & & 80 & 57 & & 11.137 & 15.761 & 17.198 \\
\hline & & & 0.876 & & & 80 & & 2.140 & 3.127 & 501 \\
\hline & & 0.872 & 0.882 & 0.863 & 0.078 & 0.056 & 0.051 & 11.157 & 15.642 & 17.077 \\
\hline & & 54 & 0.855 & 0.874 & & 0.272 & 0.247 & 2.291 & 3.143 & 3.538 \\
\hline & & 0.863 & 0.888 & 0.866 & 75 & 0.055 & 0.050 & 11.478 & 16.170 & 17.448 \\
\hline & & 0.863 & 0.871 & 0.887 & 89 & 0.277 & 0.251 & 2.217 & 3.139 & 3.529 \\
\hline & & 0.877 & 0.880 & 0.867 & 0.079 & 0.057 & 0.051 & 11.070 & 15.548 & 17.103 \\
\hline \multirow{8}{*}{$\begin{array}{c}\rho_{1}=0.5 \\
\& \\
\rho_{1}=0.9\end{array}$} & $\mathrm{CA}$ & 0.879 & 0.887 & 0.884 & 0.403 & 0.284 & 0.252 & 2.180 & 3.121 & 3.503 \\
\hline & & 0.880 & 0.883 & 0.882 & 0.726 & 0.509 & & 1.212 & 1.734 & 1.927 \\
\hline & & 0.8 & 0.874 & & 0.401 & & & 2.170 & 3.087 & 3.503 \\
\hline & & 0.865 & 0.879 & & 0.712 & 0.503 & & 1.215 & 1.746 & 1.930 \\
\hline & & & 0.872 & & & & & 2.283 & 3.179 & 3.566 \\
\hline & & & 0.870 & & & & & 1.267 & 1.765 & 1.961 \\
\hline & & & 0.882 & & 4 & 0 . & 49 & 2.213 & 3.149 & 3.530 \\
\hline & & & 0.8 & & 20 & & & 1.201 & 1.749 & 1.928 \\
\hline \multirow{8}{*}{$\begin{array}{c}\rho_{1}=0.9 \\
\& \\
\rho_{1}=0.1\end{array}$} & & 58 & 0.891 & & 11 & 0.510 & & 1.207 & 1.747 & 1.905 \\
\hline & & 84 & 0.879 & 84 & 79 & 0.056 & 51 & 11.168 & 15.658 & 17.250 \\
\hline & & & 0.884 & & 07 & 0.508 & & 1.207 & 1.740 & 1.893 \\
\hline & $\mathrm{T} 2$ & 0.869 & 0.863 & 0.877 & 0.078 & 0.056 & 0.051 & 11.196 & 15.531 & 17.248 \\
\hline & & 0.839 & 0.877 & 0.864 & 0.665 & 0.493 & 0.445 & 1.262 & 1.779 & 1.943 \\
\hline & BB2 & 0.863 & 0.867 & 0.879 & 0.074 & 0.054 & 0.050 & 11.601 & 15.978 & 17.653 \\
\hline & & 0.856 & 0.878 & 0.869 & 0.694 & 0.503 & 0.453 & 1.233 & 1.746 & 1.920 \\
\hline & & 0.864 & 0.870 & & 0.078 & 0.056 & 0.051 & 11.010 & 15.602 & 17.311 \\
\hline \multirow{8}{*}{$\begin{array}{c}\rho_{1}=0.9 \\
\& \\
\rho_{1}=0.5\end{array}$} & & 0.858 & 0.894 & & 17 & 0.513 & 0.457 & 1.197 & 1.742 & 1.913 \\
\hline & & & & & & 86 & & 2.104 & 3.128 & 3.467 \\
\hline & & & 0.888 & & & & & & 1.735 & 920 \\
\hline & VST2 & 0.829 & 0.888 & 0.885 & 0.395 & 0.283 & 0.254 & 2.097 & 3.138 & 3.487 \\
\hline & BB1 & 0.835 & 0.881 & & 0.668 & 0.495 & 0.444 & 1.250 & 1.781 & 1.936 \\
\hline & BB2 & 0.825 & 0.885 & 0.876 & 0.378 & 0.276 & 0.249 & 2.181 & 3.208 & 3.516 \\
\hline & PB1 & 0.838 & 0.891 & 0.873 & 0.699 & 0.506 & 0.452 & 1.199 & 1.761 & 1.931 \\
\hline & PB2 & 0.827 & 0.887 & 0.885 & 0.400 & 0.284 & 0.255 & 2.068 & 3.124 & 3.473 \\
\hline
\end{tabular}

Stat., Optim. Inf. Comput. Vol. 2, March 2014. 
Table-6 : Simulation results of coverage percentage, average lengths and relative coverage for $90 \%$ confidence intervals of queueing Network $E_{4} / H_{4}^{P o} / 1$ to

$$
H_{4}^{P o} / E_{4} / 1
$$

\begin{tabular}{|c|c|c|c|c|c|c|c|c|c|c|}
\hline \multirow{2}{*}{$\begin{array}{c}\text { Intensity } \\
\text { Parameters }\end{array}$} & \multirow{2}{*}{$\begin{array}{l}\text { Estimation } \\
\text { Approches }\end{array}$} & \multicolumn{3}{|c|}{ Coverage Percentages } & \multicolumn{3}{|c|}{ Average Lengths } & \multicolumn{3}{|c|}{ Relative Coverage } \\
\hline & & \multicolumn{9}{|c|}{$n=10|n=20| n=25|n=10| n=20|n=25| n=10 \mid n=20 n=25$} \\
\hline \multirow{8}{*}{$\begin{array}{c}\rho_{1}=0.1 \\
\stackrel{\&}{=} \\
\rho_{1}=0.5\end{array}$} & CAN1 & 0.881 & 0.882 & 0.885 & & & & 11.120 & 15.505 & 17.401 \\
\hline & CAN2 & 0.864 & 0.868 & 0.898 & 0.397 & 0.283 & 0.253 & 2.174 & 3.063 & 3.549 \\
\hline & VST1 & 0.872 & 0.881 & 0.873 & 0.079 & 0.057 & 0.051 & 11.037 & 15.557 & 17.232 \\
\hline & VST2 & 0.852 & 0.863 & 0.891 & 0.390 & 0.280 & 0.251 & 2.185 & 3.078 & 3.551 \\
\hline & BB1 & 858 & 0.871 & 0.868 & 0.074 & 0.055 & 0.049 & 11.590 & 15.867 & 17.596 \\
\hline & BB2 & .845 & 0.858 & 0.890 & 0.374 & 0.274 & 0.246 & 2.258 & 3.135 & 3.616 \\
\hline & PB 1 & .874 & 0.880 & 0.875 & 0.077 & 0.056 & 0.050 & 11.289 & 15.698 & 17.423 \\
\hline & PB2 & 0.851 & 0.857 & 0.884 & 0.394 & 0.281 & 0.251 & 2.159 & 3.050 & 3.516 \\
\hline \multirow{8}{*}{$\begin{array}{c}\rho_{1}=0.1 \\
\stackrel{\&}{=} \\
\rho_{1}=0.9\end{array}$} & CAN1 & 0.871 & 0.882 & 0.884 & 0.081 & 0.057 & 0.051 & 10.762 & 15.445 & 17.311 \\
\hline & N2 & 876 & 0.886 & 0.911 & 0.707 & 0.515 & 0.454 & 1.240 & 1.720 & 2.004 \\
\hline & VST1 & 0.846 & 0.858 & 0.872 & 0.081 & 0.057 & 0.051 & 10.489 & 15.098 & 17.141 \\
\hline & VST2 & 364 & 0.880 & 0.900 & 0.692 & 0.509 & 0.450 & 1.248 & 1.729 & 1.998 \\
\hline & & 0.842 & 0.862 & 0.872 & 0.076 & 0.055 & 0.050 & 11.120 & 15.663 & 17.570 \\
\hline & 2 & 0.847 & 0.872 & 0.903 & 0.665 & 0.497 & 0.441 & 1.273 & 1.755 & 2.047 \\
\hline & & & 0.867 & 0.875 & 0.079 & 0.056 & 0.050 & 10.822 & 15.421 & 17.365 \\
\hline & PB2 & 373 & 0.881 & 0.900 & 0.700 & 0.510 & 0.452 & 1.247 & 1.726 & 1.992 \\
\hline \multirow{8}{*}{$\begin{array}{c}\rho_{1}=0.5 \\
\& \\
\rho_{1}=0.1\end{array}$} & & .864 & 0.869 & $\overline{9}$ & .399 & 0.284 & 0.257 & 2.163 & 3.057 & 3.541 \\
\hline & & + & 0.875 & & & & & 11.043 & 15.273 & 17.334 \\
\hline & & 6 & 0.864 & & 0. & & & 2.128 & 3.053 & 3.521 \\
\hline & $\mathrm{T} 2$ & 355 & 0.873 & 0.877 & 0.078 & 0.057 & 0.050 & 11.008 & 15.429 & 17.411 \\
\hline & & 36 & 0.859 & 0.898 & 0.373 & 0.275 & 0.249 & 2.239 & 3.129 & 3.603 \\
\hline & & & 0.861 & 0.874 & 0.074 & 0.055 & 0.049 & 11.492 & 15.567 & 17.692 \\
\hline & & & 0.862 & 0.897 & 0.390 & 0.280 & 0.253 & 2.160 & 3.080 & 3.543 \\
\hline & 2 & 0.858 & 0.864 & 0.870 & 0.079 & 0.057 & 0.051 & 10.895 & 15.197 & 17.222 \\
\hline \multirow{8}{*}{$\begin{array}{c}\rho_{1}=0.5 \\
\& \\
\rho_{1}=0.9\end{array}$} & CAN1 & 0.874 & 0.891 & 0.887 & 0.397 & 0.281 & 0.254 & 2.200 & 3.169 & 3.486 \\
\hline & & & 0.880 & 0.880 & 0.722 & 0.513 & & 1.216 & 1.717 & 1.924 \\
\hline & & & 0.879 & 0.882 & 0.396 & 0.280 & 0.254 & 2.161 & 3.142 & 3.472 \\
\hline & & & 0.853 & 0.870 & 0.707 & 0.506 & 0.452 & 1.230 & 1.685 & 1.923 \\
\hline & & & 0.880 & 0.875 & 0.372 & 0.272 & 0.247 & 2.287 & 3.241 & 3.538 \\
\hline & & 860 & 0.863 & 0.873 & 0.679 & 0.495 & 0.445 & 1.267 & 1.742 & 1.964 \\
\hline & & & 0.883 & 0 . & 0.388 & 0.277 & 0.2 & 2.185 & 3.188 & 3.478 \\
\hline & & & 0.8 & & 0.716 & 08 & & 1.206 & 1.680 & 1.924 \\
\hline \multirow{8}{*}{$\begin{array}{c}\rho_{1}=0.9 \\
\& \\
\rho_{1}=0.1\end{array}$} & & 57 & 0.890 & & 12 & 0.517 & & 1.203 & 1.721 & 1.897 \\
\hline & & & 0.881 & 80 & 81 & 0.057 & & 10.803 & 15.503 & 17.302 \\
\hline & & & 0.868 & 0.861 & 0.708 & 0.515 & & 1.196 & 1.685 & 1.885 \\
\hline & VST2 & 0.840 & 0.867 & 0.886 & 0.079 & 0.056 & 0.050 & 10.617 & 15.426 & 17.580 \\
\hline & & 0.838 & 0.880 & 0.865 & 0.666 & 0.499 & 0.445 & 1.259 & 1.764 & 1.945 \\
\hline & & 0.854 & 0.868 & 0.874 & 0.076 & 0.055 & 0.049 & 11.223 & 15.844 & 17.692 \\
\hline & & 0.851 & 0.870 & 0.867 & 0.694 & 0.509 & 0.453 & 1.225 & 1.708 & 1.913 \\
\hline & & 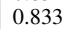 & 0.870 & & 0.080 & 0.056 & 0.051 & 10.389 & 15.422 & 17.480 \\
\hline \multirow{8}{*}{$\begin{array}{c}\rho_{1}=0.9 \\
\& \\
\rho_{1}=0.5\end{array}$} & & 0.865 & 0.889 & 0.904 & 0.709 & 0.509 & 0.457 & 1.220 & 1.745 & 1.979 \\
\hline & & & 0.8 & & 0.401 & 0.286 & & 2.158 & 3.089 & 3.552 \\
\hline & & 0 . & 0.880 & & 0.706 & 0.507 & 0.455 & 1.193 & 1.734 & 1.958 \\
\hline & VST2 & 0.843 & 0.878 & 0.897 & 0.393 & 0.282 & 0.249 & 2.147 & 3.109 & 3.596 \\
\hline & & 0.838 & 0.880 & & 0.663 & 0.492 & 0.444 & 1.265 & 1.790 & 2.013 \\
\hline & BB2 & 0.834 & 0.873 & 0.888 & 0.377 & 0.276 & 0.245 & 2.213 & 3.165 & 3.629 \\
\hline & PB1 & 0.848 & 0.886 & 0.894 & 0.691 & 0.502 & 0.451 & 1.227 & 1.764 & 1.981 \\
\hline & PB2 & 0.840 & 0.880 & 0.894 & 0.397 & 0.284 & 0.250 & 2.114 & 3.104 & 3.574 \\
\hline
\end{tabular}

Note that:

1. boldface denotes the greatest relative coverage among estimation approaches.

2. confidence intervals for $\rho_{1}$ under different estimation approaches are denoted by CAN1, VST1, BB1, PB1 and that of $\rho_{2}$ are denoted by CAN2, VST2, BB2, PB2. 


\section{REFERENCES}

1. Burke P.J.(1956): Output of a Queueing System, Operations Research, Vol.4, pp.175-178.

2. Disney R. L.(1975): Random flow in queueing networks: a review and a critique. Trans. A.I.E.E., 7, pp. 268-288.

3. Efron B.(1979): Bootstrap methods; another look at the jackknife. Annals of Statistics 7, pp.126.

4. Efron B.(1982): The jackknife, the bootstrap, and other resampling plans. SIAM Monograph \#38.

5. Efron B.(1987): Better bootstrap confidence intervals. J Am Stat Assoc 82, pp.171- 200. doi: $10.2307 / 2289144$

6. Gross D. and Harris C.M.(1998): Fundamentals of Queueing Theory. 3rd Ed New York: John Wiley.

7. Hogg R.V. and Craig A.T. (1995): Introduction to Mathematical Statistics. Prentice- Hall, Inc.

8. Jackson J. R.(1957): Networks of Waiting Lines, Operations Research 5, pp. 518- 21.

9. Ke J. C. and Chu Y. K. (2009): Comparison on five estimation approaches of intensity for a queueing system with short run, Computational Statistics, Vol. 24 Issue-4, pp. 567-582, Springer-Verlag.

10. Ke J.C. and Chu Y.K. (2006): Nonparametric and simulated analysis of intensity for queueing system. Applied Mathematics and Computation, 183. pp. 1280-1291.

11. Kleinrock L.(1976): Queueing Systems, Vol. II, Computer Applications, John Wiley \& Sons, New York.

12. Rubin D.B. (1981): The Bayesian bootstrap. The Annals of Statistics 9, pp. 130-134.

13. Thiruvaiyaru D. and Basava I.V.(1996): Maximum likelihood estimation for queueing networks, In Stochastic Processes and Statistical Inference (Eds., B.L.S. Prakasa Rao and B. R. Bhat),(1996), pp.132-149, New Age International Publications, New Delhi.

14. Thiruvaiyaru D., Basava I.V. and Bhat U. N. (1991): Estimation for a class of simple queueing network. Queueing Systems 9, pp. 301-312. 